\title{
SPECTROSCOPIC STUDIES OF THE ANAEROBIC ENZYME-SUBSTRATE COMPLEX OF CATECHOL 1,2-DIOXYGENASE
}

Geoff P. Horsman ${ }^{*}, 1$, Andrew Jirasek ${ }^{*, 2,3, \dagger}$, Frédéric H. Vaillancourt ${ }^{1, \dagger}$, Christopher J. Barbosa $^{2,3, \dagger}$, Andrzej A. Jarzecki ${ }^{4, \dagger}$, Changliang Xu ${ }^{4}$, Yasmina Mekmouche ${ }^{5, \dagger}$, Thomas G. Spiro $^{4}$, John D. Lipscomb ${ }^{5}$, Michael W. Blades ${ }^{2}$, Robin F.B. Turner ${ }^{3,6}$, and Lindsay D. Eltis $^{1, * *}$

${ }^{1}$ Departments of Biochemistry and Microbiology, The University of British Columbia, Vancouver, BC, V6T 1Z3, Canada. ${ }^{2}$ Department of Chemistry, The University of British Columbia, Vancouver, BC, V6T 1Z1, Canada. ${ }^{3}$ Michael Smith Laboratories, The University of British Columbia, Vancouver, BC, V6T 1Z4, Canada. ${ }^{4}$ Department of Chemistry, Princeton University, Princeton, NJ, 08544, USA. ${ }^{5}$ Department of Biochemistry, Molecular Biology and Biophysics, University of Minnesota, Minneapolis, MN, 55455, USA. ${ }^{6}$ Department of Electrical and Computer Engineering, The University of British Columbia, Vancouver, BC, V6T 1Z4, Canada. 'Present addresses: AJ: Dept. of Physics and Astronomy, The University of Victoria, Victoria, BC, V8W 3P6, Canada; FHV: Dept. of Biological Chemistry and Molecular Pharmacology, Harvard Medical School, Boston, MA, 02115, USA; CJB: Inex Pharmaceuticals, Burnaby, BC, V5J 5J8, Canada; AAJ: Department of Chemistry, Brooklyn College and the Graduate School of the City University of New York, Brooklyn, NY, 11230, USA; YM: Faculté des Sciences de Saint Jérôme, case 432, Avenue Escadrille Normandie-Niemen, 13397 Marseille Cedex 20, France

*** Corresponding author: Lindsay D. Eltis, leltis@interchange.ubc.ca 


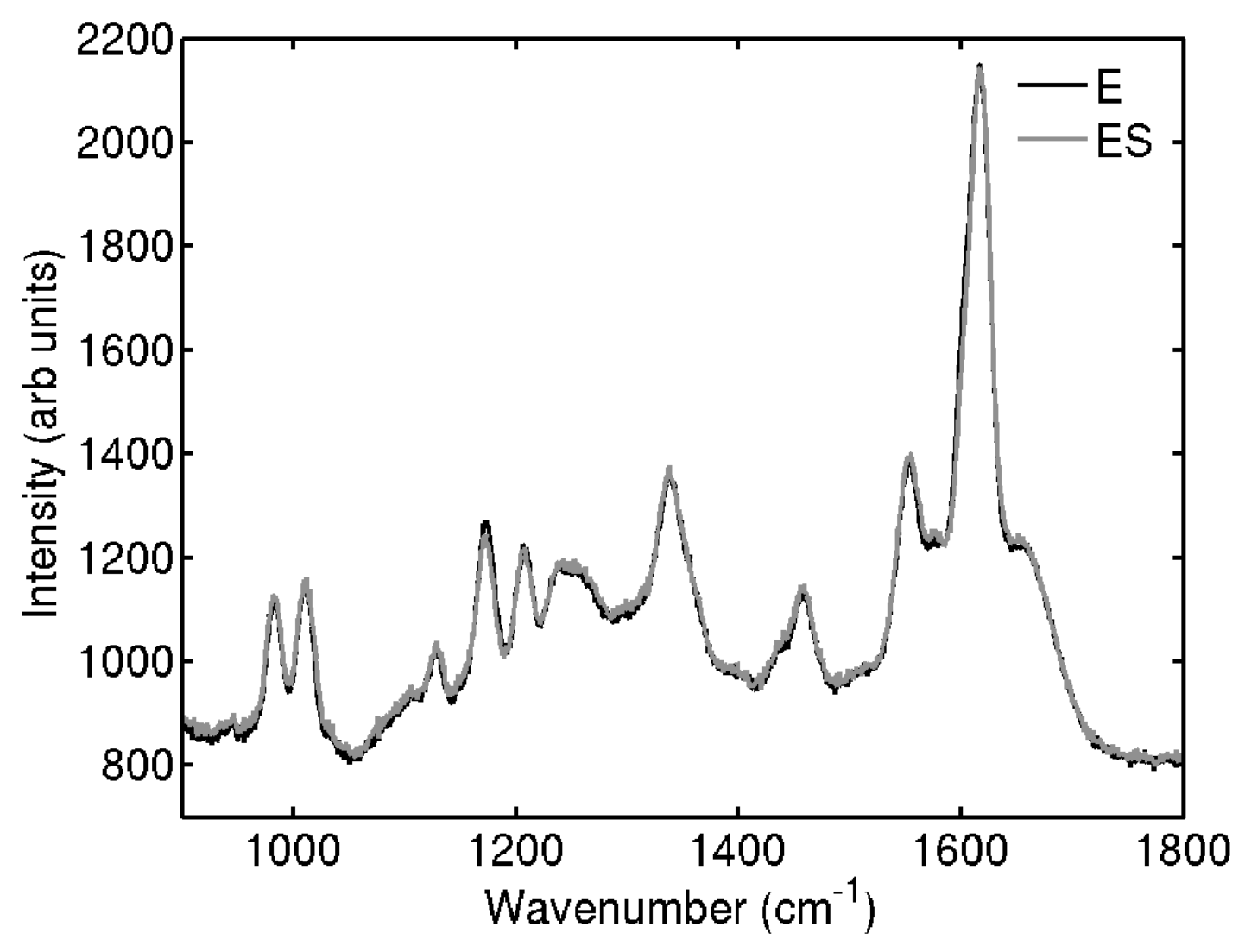

Figure S1. Overlay of free C12O enzyme (E) and C12O:catechol complex (ES) UVRR spectra that were used to construct the difference spectrum in Figure 4a. 